\title{
A Novel Contact Tunnel Profile Monitoring System: Concept and Application
}

\author{
Yang Han, ${ }^{1,2}$ Shikun Pu, ${ }^{1}$ Lei Gao, ${ }^{1}$ Jianli Duan, ${ }^{1}$ and Erbing Li $\mathbb{D}^{3}$ \\ ${ }^{1}$ College of Defense Engineering, Army Engineering University of PLA, Nanjing, Jiangsu 210007, China \\ ${ }^{2}$ Nanjing Urban Construction Management Group Co., Ltd., Nanjing, Jiangsu 210006, China \\ ${ }^{3}$ College of Field Engineering, Army Engineering University of PLA, Nanjing, Jiangsu 210007, China
}

Correspondence should be addressed to Erbing Li; lebingest@126.com

Received 29 October 2020; Revised 13 November 2020; Accepted 12 December 2020; Published 19 December 2020

Academic Editor: Hao Wang

Copyright (C) 2020 Yang Han et al. This is an open access article distributed under the Creative Commons Attribution License, which permits unrestricted use, distribution, and reproduction in any medium, provided the original work is properly cited.

\begin{abstract}
The displacement of the cross section directly reflects the stress state and stability of the surrounding rock and structure, so the monitoring of it is essential during the construction and operation of the tunnel and underground engineering, particularly under the conditions of earthquake and other geological disasters. This paper introduces a new contact tunnel profile monitoring system (TPMS) in detail that uses a tilt sensor and a displacement sensor as data acquisition devices. According to the relation between the sensing physical quantity and displacement change, the displacement calculation formulas of the tunnel cross section measuring points based on the two-dimensional plane coordinate system were deduced, and in order to eliminate the actual installation and positioning deviation of the monitoring system, the method of obtaining the optimal monitoring plane and converting coordinates of the measuring points was proposed, thus establishing the theoretical basis for the application of the TPMS. With the Beishan exploration tunnel (BET) in China as the test platform, the TPMS was successfully applied for long-term monitoring. The application experience showed that the TPMS can realize continuous monitoring, automatic collection and transmission of the monitoring data, remote access whenever necessary, without affecting the transportation in the tunnel, and high accuracy, which reaches $0.01 \mathrm{~mm}$. This system provides a new simple and effective method with good generality and applicability for the deformation monitoring of the tunnel and underground engineering.
\end{abstract}

\section{Introduction}

During the construction and operation of the tunnel and underground engineering, it is crucial to guarantee the safety and stability of the surrounding rock and structure. The convergence displacement of the surrounding rock directly reflects the stability of a rock mass after excavation $[1,2]$ and also provides support for the inversion of the rock mechanics parameters and the determination of a reasonable support time. Therefore, convergence displacement monitoring of the surrounding rock and structure is an essential item for underground engineering deformation monitoring, and the applicability of monitoring methods and systems is of vital importance to guaranteeing safety during tunnel and underground engineering construction and operation.
At present, the monitoring methods for the convergence displacement of the surrounding rock are mainly divided into two categories: noncontact and contact monitoring. Noncontact monitoring methods mainly include the automatic tracking total station measurement method, three-dimensional (3D) laser scanning, and closerange photography [3-9]. The above researchers have conducted many studies with respect to the monitoring principle, the feasibility of monitoring, and the proposal of monitoring programs $[10,11]$. The advantages of the total station method are the high measurement accuracy and low light requirements during the measurement process; however, this method is easily affected by the dust generated in the construction process, and the layout of the total station in the full-length of the tunnel is relatively 
expensive. The advantages of 3D scanning are fast measurement speed, complete and accurate measurement data, and low light requirements for the test environment, but the registration, denoising, splicing of the point cloud data obtained, and slice after 3D modeling still have errors and reliability issues. The advantage of close-range photography is that the measurement is convenient and fast, but the measurement accuracy is greatly affected by the lighting condition. Specifically, the light source cannot work when light is limited, so an artificial light source should be used as a supplement. The above noncontact measurement methods are mainly based on optical principles, and its measurement accuracy can only reach the millimeter level. For real-time monitoring in long-term health diagnosis [12], the system equipment needs to be fixed near the center of the tunnel section, which will affect normal operation.

Contact monitoring methods that are used to measure the convergence displacement mainly include a convergence meter and a convergence measurement system [13-16]. The convergence meter is a measurement instrument specially used to monitor the distance change (i.e., convergence value) between any two points on the wall of the tunnel or other engineering surrounding rock. This instrument has a digital display and vibrating-wire convergence meter and is widely used to measure the convergence deformation of tunnels and underground engineering. The Bassett convergence system (BCS) is a representative convergence measurement system that uses a tilt sensor and a long and short arm structure to transfer the displacement between measuring points in order to obtain the relative displacement between these points. With the development of optical fiber technology, in recent years, some scholars have used a fiber Bragg grating for convergence displacement contact measurements $[17,18]$. Among them, the convergence meter has now realized automatic measurements with an accuracy reaching $0.01 \mathrm{~mm}$, but there are some problems, such as the large interference effects in the construction and operation of the project. The BCS has high precision and automation, but it is fixed and not suitable for large-scale layouts because of high cost. As a new method for measuring the convergence displacement of surrounding rock, fiber Bragg gratings are not yet mature, with some issues, such as difficult laying and obvious influence of temperature change.

In terms of the disadvantages of the methods mentioned above, a new-type contact tunnel profile monitoring system (TPMS) was developed to monitor the long-term deformation and performance of the tunnel surrounding rock and structure. The operating principle of this monitoring system is similar to that of the BCS; that is, it can realize the automation and continuity of the measurement process. Additionally, this system is simple in structure, relatively cheap, suitable for different sections, and reusable.

\section{Basic Principles of the TPMS}

2.1. Composition of the TPMS. The TPMS is composed of a series of linked arms that are fixed to the tunnel wall. Each arm is fitted with a high-accuracy displacement sensor and precision tilt sensor to monitor the deformation, and a data logging system is available to automatically collect the realtime displacement and transmit it to a computer. A typical measurement unit of the TPMS is shown in Figure 1, including the sensor, rod end, extension tube, and fixed anchor. The length of the measuring arm between two adjacent measuring points can be adjusted on the spot using extension tubes. As an independent working part, the number of TPMS measurement units can be increased and decreased according to the size of the tunnel section and the number of measuring points. The TPMS can be applied to various section forms and can be set up on unsupported bare rock. In addition, the system can be modularly assembled. After the measurement unit is disassembled, it can be reused in many cases except for the fixed anchor.

The sensors of the TPMS include a tilt sensor and a displacement sensor (Figure 2). The tilt sensor is a capacitive sensor, the displacement sensor is a resistive sensor, and the main specifications of the tilt and displacement sensors are shown in Table 1. Both of these sensors produce a signal that can be converted to a change in the microvoltage according to the change in the physical quantity of the sensor. The data logging system of the TPMS is composed of a data collector and data processing software. The data collector model is CR800 produced by Campbell Scientific company, which is a research-level data collector characterized by low cost, small size, and different channels (Figure 3). When TPMS monitoring is conducted, the transducer transfers signals to the data collector, which is connected to the remote-control computer using an RS-232 communication mode. Using the measurement and control software, automatic real-time monitoring can be realized.

As shown in Figure 4, the TPMS is available in either an open or closed loop structure around the inside of the tunnel. Compared with a closed loop structure, an open loop structure omits the linked measuring arm AH. The closed loop structure is analogous to that of conventional closed end survey techniques, while the open loop structure must be referenced to a reference point. Variations from the relative displacements between adjacent measuring points are measured, and after they are transmitted to the reference point, the displacement variations at each measuring point relative to the reference point can be obtained.

2.2. Calculation Principle for the TPMS. A position change between adjacent measuring points will cause the length and angle of the measuring arm to change. Through the displacement and tilt sensors, the change in the length and angle can be obtained, giving the relative displacement between the measuring points. With any measuring point on the monitoring section taken as a reference point, the relative displacement of each measuring point relative to the reference point can be obtained through recursion. Generally, the engineering datum point is located in a region with stable deformation, and it is believed that the displacement caused by that is negligible. The relative displacements of the two measuring points are obtained by the sensor variation values between the two measuring points, and the absolute displacement of each measuring point 


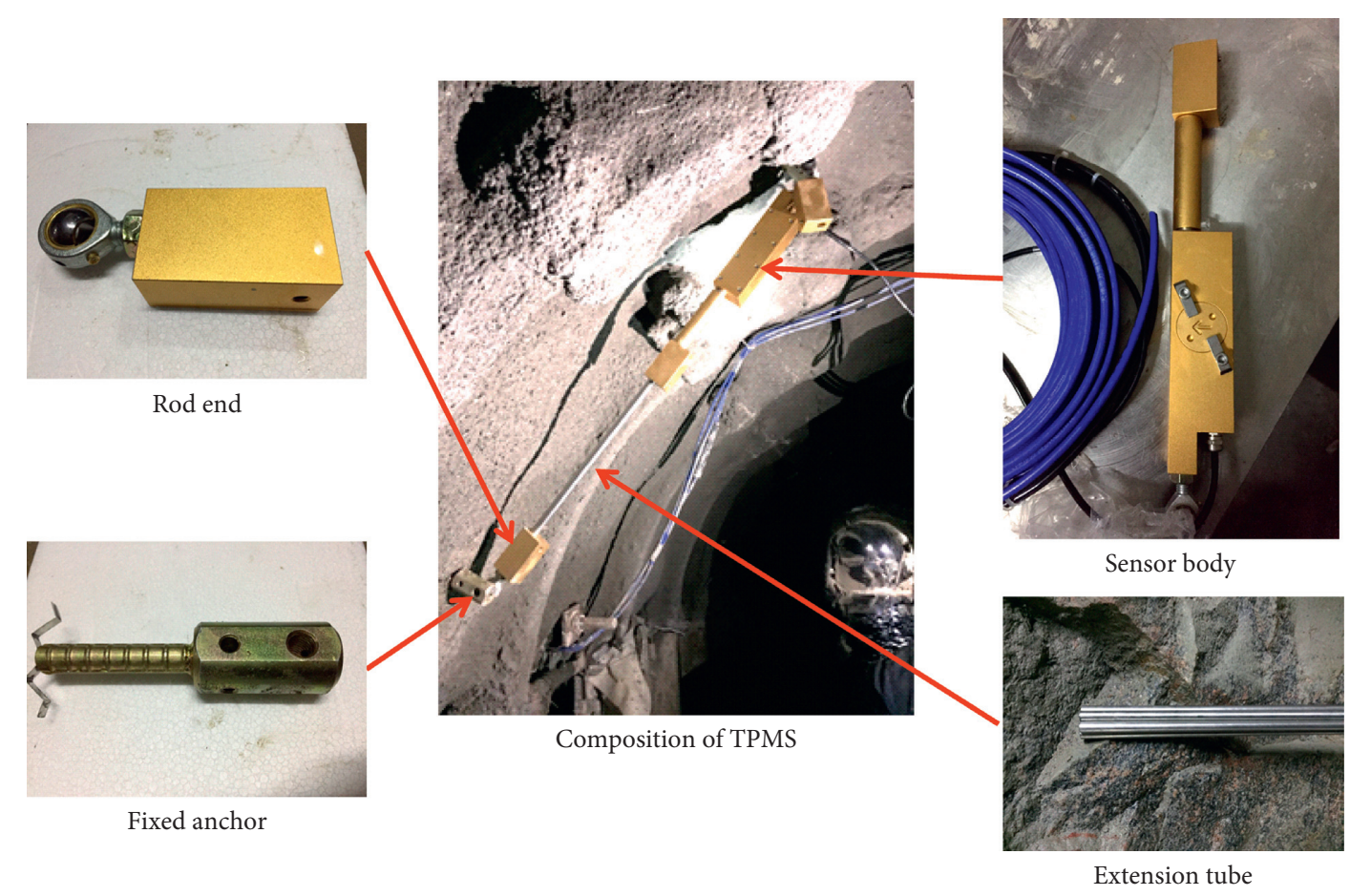

Figure 1: Typical measurement unit of the TPMS.

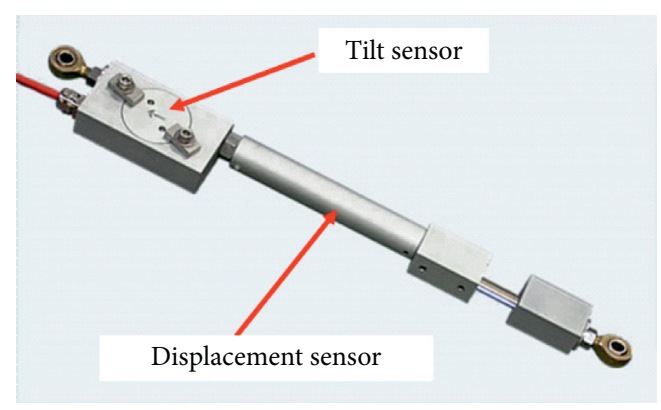

Figure 2: Composition of the TPMS sensor.

TABle 1: Main technical specifications of TPMS sensors.

\begin{tabular}{lcc}
\hline Specification & Tilt sensor & Displacement sensor \\
\hline Manufacturer & VI Tec (USA) & Honeywell (USA) \\
Sensor model & MEMS(micro-electro-mechanical systems) & MLT-38000101 \\
Range & $\pm 5^{\circ}$ & $0 \sim 50 \mathrm{~mm}$ \\
Accuracy & $0.003^{\circ}$ & $0.01 \mathrm{~mm}$ \\
Input voltage & $5.5 \sim 15 \mathrm{Vdc}$ & $5.5 \sim 15 \mathrm{Vdc}$ \\
Output voltage & $\pm 2.5 \mathrm{Vdc}$ & $\pm 2.5 \mathrm{Vdc}$ \\
Operating temperature & $-40 \sim+85^{\circ} \mathrm{C}$ & $-40 \sim+80^{\circ} \mathrm{C}$ \\
\hline
\end{tabular}

relative to the engineering datum point is obtained via calculation.

It is assumed that the measuring points are serially numbered to $0,1,2, \ldots, n$ starting from the reference point. Under the global coordinate system for the monitoring plane, the initial coordinate for each measuring point is $\left(x_{0,0}^{G}\right.$, $\left.y_{0,0}^{G}\right),\left(x_{1,0}^{G}, y_{1,0}^{G}\right),\left(x_{2,0}^{G}, y_{2,0}^{G}\right), \ldots,\left(x_{i, j}^{G}, y_{i, j}^{G}\right), i, j=0,1,2, \ldots, n$ (Figure 5). Superscript $G$ on the coordinates denotes the global coordinate system; the first subscript $i$ denotes the serial number of the measuring points; the second subscript $j$ denotes the measurement times; and 0 denotes the initial position of measuring points. In the local coordinate system of adjacent measuring points, the initial coordinate of each measuring point is $\left(x_{0,0}^{L}, y_{0,0}^{L}\right),\left(x_{1,0}^{L}, y_{1,0}^{L}\right),\left(x_{2,0}^{L}, y_{2,0}^{L}\right), \ldots$, $\left(x_{i, j}^{L}, y_{i, j}^{L}\right), i, j=0,1,2, \ldots, n$. The superscript $L$ on the coordinates denotes the local coordinate system, and the 


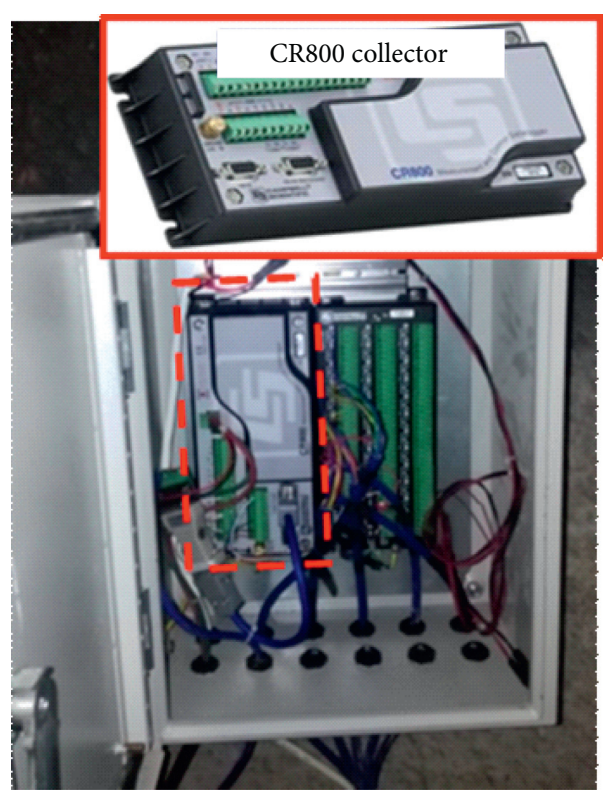

FIgURE 3: Data collection box.

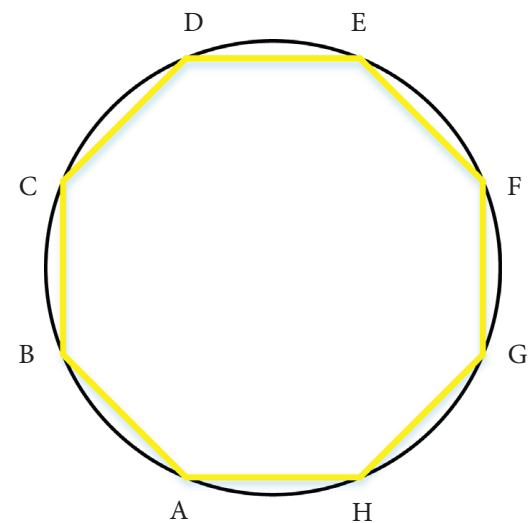

(a)

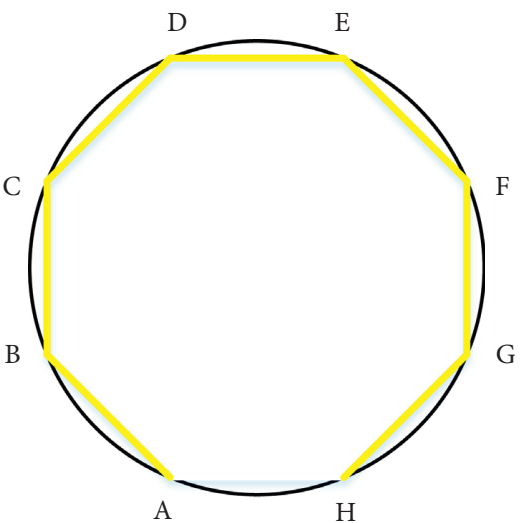

(b)

FIgURE 4: Schematic diagram of the TPMS monitoring configurations: (a) closed loop structure; (b) open loop structure.

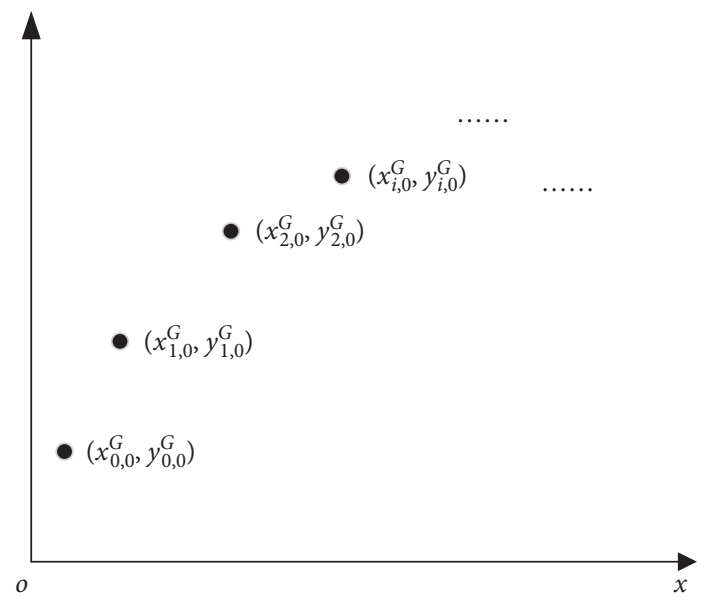

FIgURE 5: Initial coordinates of each measuring point in the global coordinate system. 
subscripts are the same as above. The main symbols and their basic meanings in the text are shown in abbrevations section.

To analyze the relative displacement between adjacent measuring points, a random measuring point $i-1$ is taken to build a local coordinate system relative to the measuring point $i$ (Figure 6). During the actual measurement, the measuring points $i-1$ and $i$ both move, so the relative position of the measuring arm at any time can be obtained by translating the measuring arm between two points with the initial position of point $i-1$ as the origin point. In Figure 6 , the initial length of the measuring arm between point $i$ and point $i-1$ is $L_{i}$, the rotation angle of the measuring arm is $\Delta \theta_{i j}$, and the length variation is $\Delta L_{i j}$ during the $j$ th measurement.

Initial local coordinate for measuring point $i$ is as follows:

$$
\left.\begin{array}{l}
x_{i, 0}^{L}=x_{i, 0}^{G}-x_{i-1,0}^{G} \\
y_{i, 0}^{L}=y_{i, 0}^{G}-y_{i-1,0}^{G}
\end{array}\right\} .
$$

The initial length of the measuring arm is

$$
L_{i}=\sqrt{\left(x_{i, 0}^{L}\right)^{2}+\left(y_{i, 0}^{L}\right)^{2}}
$$

Horizontal and vertical coordinates for the point with the length equal to the initial length on the measuring bar under $i-1$ local coordinates are as follows:

$$
\left.\begin{array}{c}
x_{i, j}^{R}=x_{i, 0}^{L} \cos \Delta \theta_{i j}+y_{i, 0}^{L} \sin \Delta \theta_{i j} \\
y_{i, j}^{R}=-x_{i, 0}^{L} \sin \Delta \theta_{i j}+y_{i, 0}^{L} \cos \Delta \theta_{i j}
\end{array}\right\} .
$$

Thus, according to the geometrical relationship between the elongation $\Delta L_{i j}$ of the measuring bar arm and the initial measuring arm length $L_{i}$, the horizontal and vertical coordinates for measuring point $i$ during the $j$ th measurement under a local coordinate system for $i-1$ are as follows:

$$
\left.\begin{array}{c}
x_{i, j}^{L}=\frac{L_{i}+\Delta L_{i j}}{L_{i}} x_{i, j}^{R} \\
y_{i, j}^{L}=\frac{L_{i}+\Delta L_{i j}}{L_{i}} y_{i, j}^{R}
\end{array}\right\} .
$$

The global coordinate for measuring point $i$ during the jth measurement is

$$
\left.\begin{array}{l}
x_{i, j}^{G}=\frac{L_{i}+\Delta L_{i j}}{L_{i}}\left(x_{i, j}^{L} \cos \Delta \theta_{i j}+y_{i, j}^{L} \sin \Delta \theta_{i j}\right)+x_{i-1, j}^{G} \\
y_{i, j}^{G}=\frac{L_{i}+\Delta L_{i j}}{L_{i}}\left(-x_{i, j}^{L} \sin \Delta \theta_{i j}+y_{i, j}^{L} \cos \Delta \theta_{i j}\right)+y_{i-1, j}^{G}
\end{array}\right\} .
$$

By taking $i=1$, the coordinates of measuring point 1 relative to reference point 0 in the $j$ th measurement can be obtained:

$$
\left.\begin{array}{c}
x_{1, j}^{G}=x_{1, j}^{L}+x_{0, j}^{G} \\
y_{1, j}^{G}=y_{1, j}^{L}+y_{0, j}^{G}
\end{array}\right\} .
$$

When constructing the global coordinate system in the measurement section, it is often considered that the reference point does not move; that is, $x_{0,0}^{G}=x_{0,1}^{G}=\ldots=x_{0, j}^{G}$ and $y_{0,0}^{G}=y_{0,1}^{G}=\ldots=y_{0, j}^{G}$. Therefore, the global coordinates of each measuring point in the $j$ th measurement can be obtained by recurrence from measuring point 1 .

The displacement is generated by subtracting the corresponding initial global coordinates from the global coordinates obtained by each measurement:

$$
\left.\begin{array}{l}
\Delta x_{i, j}=x_{i, j}^{G}-x_{i, 0}^{G} \\
\Delta y_{i, j}=y_{i, j}^{G}-y_{i, 0}^{G}
\end{array}\right\} .
$$

Thus far, the corresponding displacement changes in each measuring point can be calculated by the initial global coordinates and the variation in the measuring arm obtained by the tilt and displacement sensors.

2.3. Calculation of the Variation in the Rotation Angle and Length of the Measuring Arm. The data logging system converts the changes in the sensor angle and displacement into electrical signals, and the conversion of the angle and displacement to an output voltage is as follows.

There is a polynomial relationship between the inclination of the tilt sensor and the output voltage as follows:

$$
\theta=C_{0}+C_{1} V_{t}+C_{2} V_{t}^{2}+C_{3} V_{t}^{3}+C_{4} V_{t}^{4}+C_{5} V_{t}^{5}
$$

where $\theta$ is the inclination of the tilt sensor; $V_{t}$ is the output voltage of the tilt sensor; and $C_{0}, C_{1}, \ldots, C_{5}$ are calibration constants for the tilt sensor.

The displacement measured by the displacement sensor has a linear relationship with its output voltage:

$$
L=K_{d} V_{d}+b,
$$

where $L$ is the displacement of the displacement sensor, $V_{d}$ is the output voltage of the displacement sensor, and $K_{d}$ and $b$ are calibration constants for the displacement sensor.

After the TPMS is installed, the initial measurement is carried out to record the initial output voltages $\left(V_{t}^{0}\right.$ and $\left.V_{d}^{0}\right)$ of the tilt and displacement sensors for each measuring arm. Then, the initial values of inclination $\left(\theta_{0}\right)$ and displacement $\left(L_{0}\right)$ are obtained according to equations (8) and (9), respectively.

After the TPMS works normally, the change in the rotation angle and length of the corresponding measuring arm can be obtained by subtracting the initial values of the inclination and displacement measured by the tilt and displacement sensors:

$$
\left.\begin{array}{l}
\Delta \theta=\theta-\theta_{0} \\
\Delta L=L-L_{0}
\end{array}\right\} .
$$




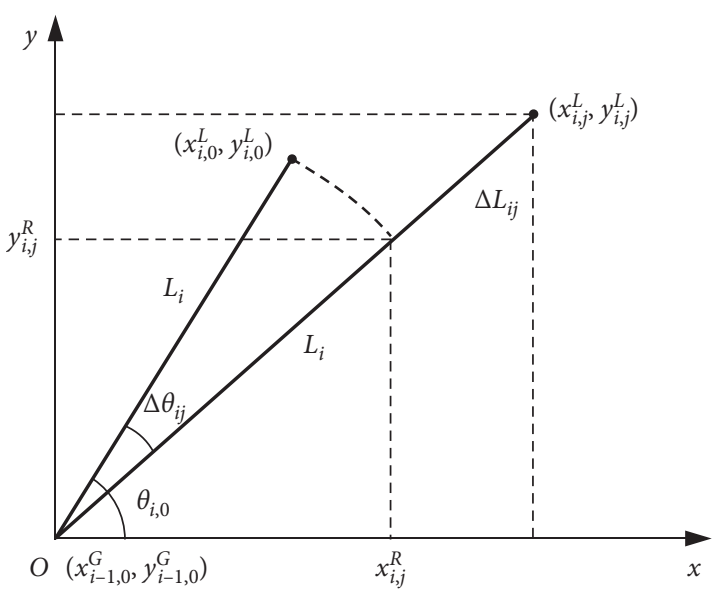

Figure 6: Local coordinate system between adjacent measuring points.

2.4. Optimization of the Monitoring Plane and 2D Coordinate Transformation. After TPMS installation, the initial coordinate for each measuring point measured using a total station is a $3 \mathrm{D}$ space coordinate under a geodetic coordinate system, which requires transformation into planar two-dimensional (2D) coordinates for monitoring the section. When fixed anchor measuring points are used, it is difficult to keep all fixed anchors in the same plane, which introduces the problem of how to determine the optimal monitoring plane.

During actual monitoring, the monitoring plane should be perpendicular to the horizontal plane. Thus, the positional relationships between the initial $3 \mathrm{D}$ space coordinates $\left(X_{i}, Y_{i}, Z_{i}\right)(i=0,1, \ldots, n)$ of each measuring point and optimal monitoring plane $\Omega$ are as follows (Figure 7).

Suppose the equation of the plane for the optimal monitoring plane is as follows:

$$
F(X, Y, Z)_{\Omega}=0 .
$$

Because the optimal monitoring plane is parallel to the $Z$-axis of the geodetic coordinate system, equation (11) can be expressed as

$$
a_{0}+a_{1} X+a_{2} Y=0
$$

where $a_{0}, a_{1}$, and $a_{2}$ are all plane equation parameters.

With the minimum sum of the distance from each measuring point to the plane, the optimized objective function is established as

$$
\min f(X, Y, Z)=\sum_{i=0}^{n} d_{i}=\sum_{i=0}^{n} \frac{\left|a_{0}+a_{1} X_{i}+a_{2} Y_{i}\right|}{\sqrt{a_{1}^{2}+a_{2}^{2}}},
$$

where $f(X, Y, Z)$ is the objective function and $d_{i}$ is the distance between measuring point $i$ and the optimal monitoring plane.

After the optimal monitoring plane is determined using the computing method above, the $3 \mathrm{D}$ spatial coordinates for measuring points need to be transformed into a $2 \mathrm{D}$ coordinate in the plane. The optimal monitoring plane is perpendicular to the horizontal plane (Figure 7). The $Y$-axis of the established $2 \mathrm{D}$ coordinate system is parallel to the $Z$ axis of the geodetic coordinate system. When the transformation of the $X$-axis and $Y$-axis coordinates for each measuring point is conducted, the points can be investigated in the $X O Y$ planes of the geodetic coordinate system (Figure 8).

The projection point of the reference point $\left(X_{0}, Y_{0}, Z_{0}\right)$ in the optimal monitoring plane is taken as the origin of the $2 \mathrm{D}$ coordinate system; thus, the $3 \mathrm{D}$ coordinate for the projection point $\left(X_{0}^{T}, Y_{0}^{T}, Z_{0}^{T}\right)$ can be obtained using the following equation:

$$
\left.\begin{array}{l}
a_{0}+a_{1} X_{0}^{T}+a_{2} Y_{0}^{T}=0 \\
\frac{X_{0}-X_{0}^{T}}{a_{1}}=\frac{Y_{0}-Y_{0}^{T}}{a_{2}} \\
Z_{0}^{T}=Z_{0}
\end{array}\right\} .
$$

According to the space vector method, the $2 \mathrm{D}$ coordinate for the projection point of any measuring point in the optimal monitoring plane is

$$
\left.\begin{array}{l}
x_{i, 0}^{G}=\frac{-a_{2}\left(X_{i}-X_{0}^{T}\right)+a_{1}\left(Y_{i}-Y_{0}^{T}\right)}{\sqrt{a_{1}^{2}+a_{2}^{2}}} \\
y_{i, 0}^{G}=Z_{i}-Z_{0}^{T}
\end{array}\right\} .
$$

\section{Practical Application of the TPMS}

3.1. Engineering Background. The new contact TPMS was successfully used in the Beishan exploration tunnel (BET). Figure 9 shows photographs of the entrance and the internal tunnel of the BET. The BET was constructed as a small pilot underground facility for the proposed underground research laboratory of the geological disposal of high-level radioactive waste in China.

The BET is located in the Jiujing site of the Beishan area, which is located approximately $80 \mathrm{~km}$ northwest of Yumen City, Gansu Province, PR China. As shown in Figure 10, the BET is mainly composed of 5 parts: a ramp (with a slope of $20^{\circ}$, a vertical depth of $50 \mathrm{~m}$, a length of $146.19 \mathrm{~m}$, a height of $2.64 \mathrm{~m}$, and a width of $2.8 \mathrm{~m}$ ), a horizontal tunnel (with an $L$ shape, a length of $110 \mathrm{~m}$, a height of $3.0 \mathrm{~m}$, and a width of $2.8 \mathrm{~m}$ ), underground test chambers (with a total length of approximately $90 \mathrm{~m}$, a height of $3.033 \mathrm{~m}$, and a width of $4.0 \mathrm{~m}$ ), a water sump, and a ventilation shaft $[19,20]$.

The surrounding rock of the BET is mainly granite with good integrity, except for the Shiyuejing Fault, and the rock of the BET is a typical hard rock with a uniaxial compressive strength above $190 \mathrm{MPa}$ [20]. The main unfavorable geology of the BET is the Shiyuejing Fault, which has an inclination of approximately $70-80^{\circ}$. The rocks in the fault zone are strongly fractured, with the various well-developed dikes. 


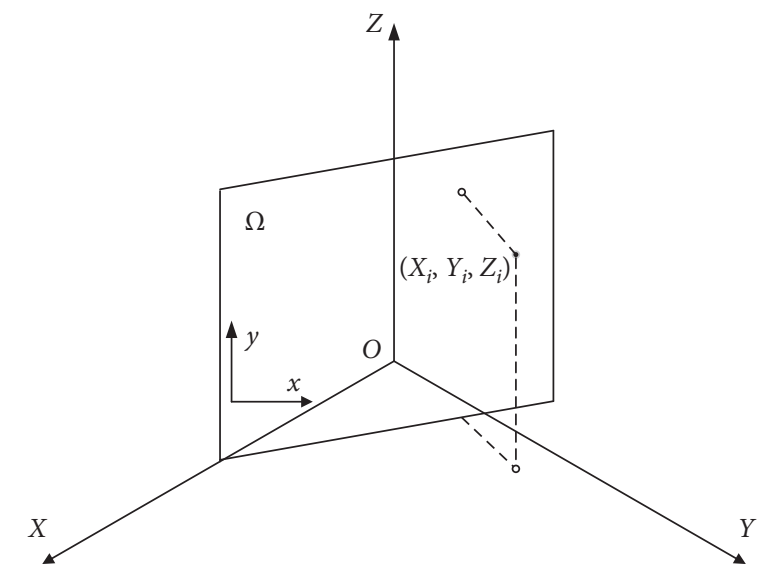

FIGURE 7: Relationships between global coordinate of measuring points and the optimal monitoring plane.

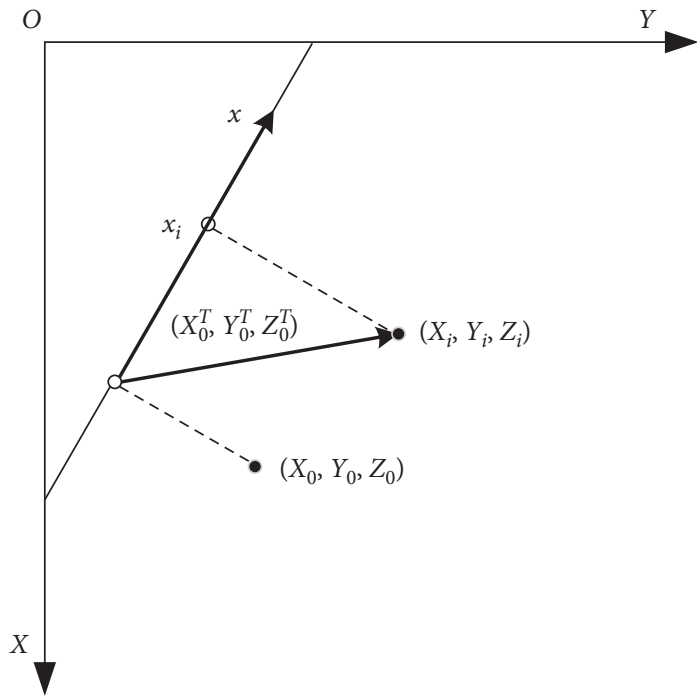

FIGURE 8: Projection plane of global coordinates and the optimal monitoring plane.

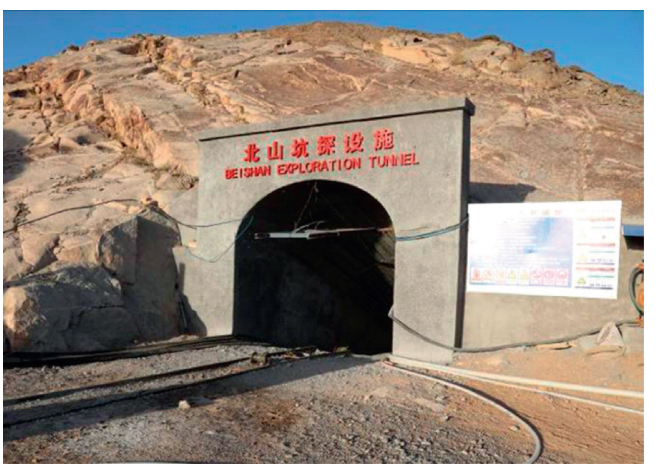

(a)

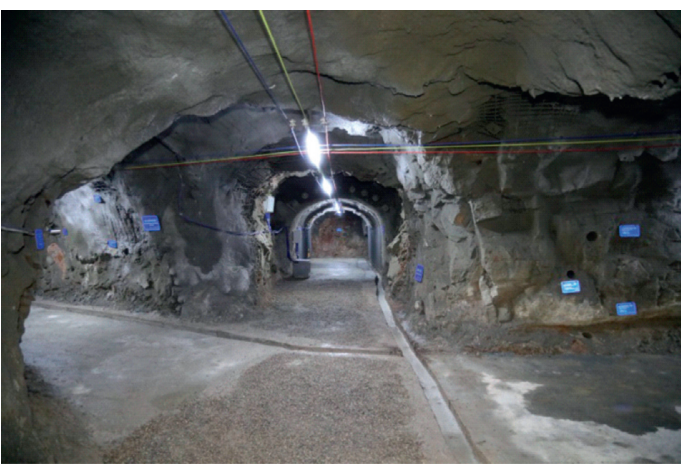

(b)

Figure 9: Photographs of the entrance and internal tunnel of the BET.

The BET was excavated by full-face smooth blasting, and it took 18 months to complete the excavation. To obtain the peripheral displacement of the BET surrounding rock, the TPMS was adopted immediately after excavation.
3.2. In Situ Measurement Scheme. The measuring section of the TPMS was set in the supporting test area of the BET, which is located in the Shiyuejing Fault zone (Figure 11). Before excavation, advanced pipe shed support in the arch 


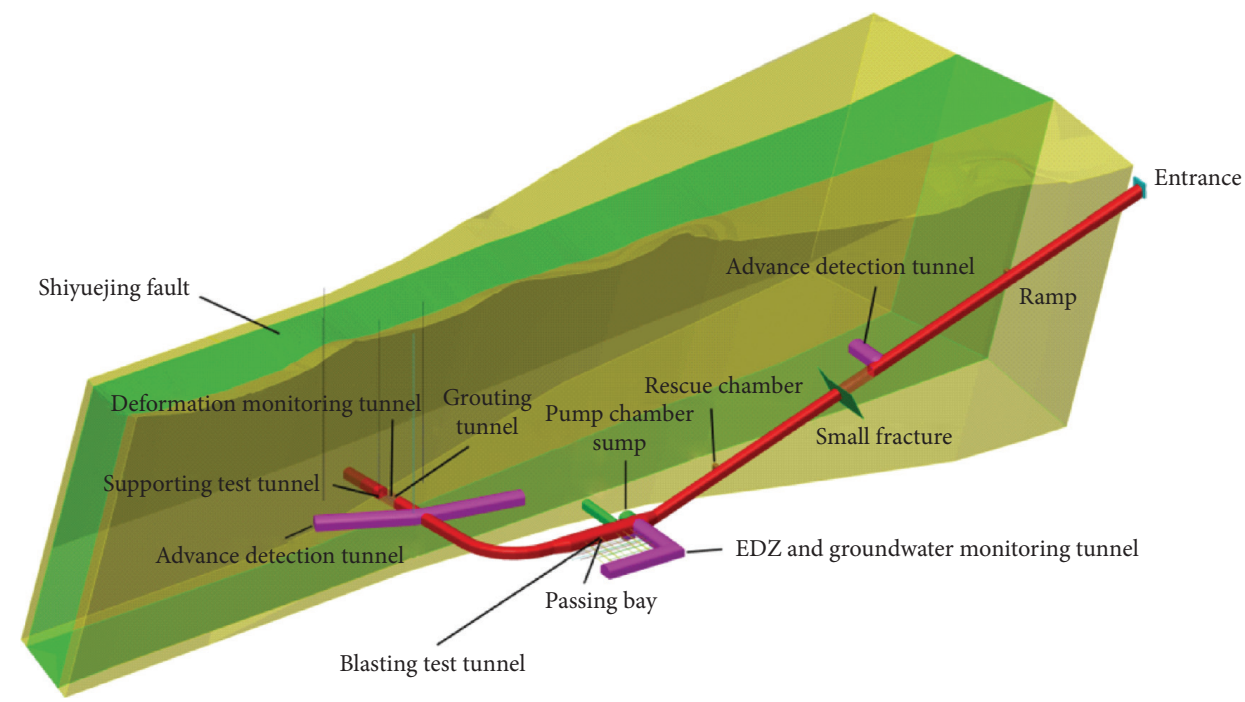

Figure 10: Spatial structure of the BET [19].
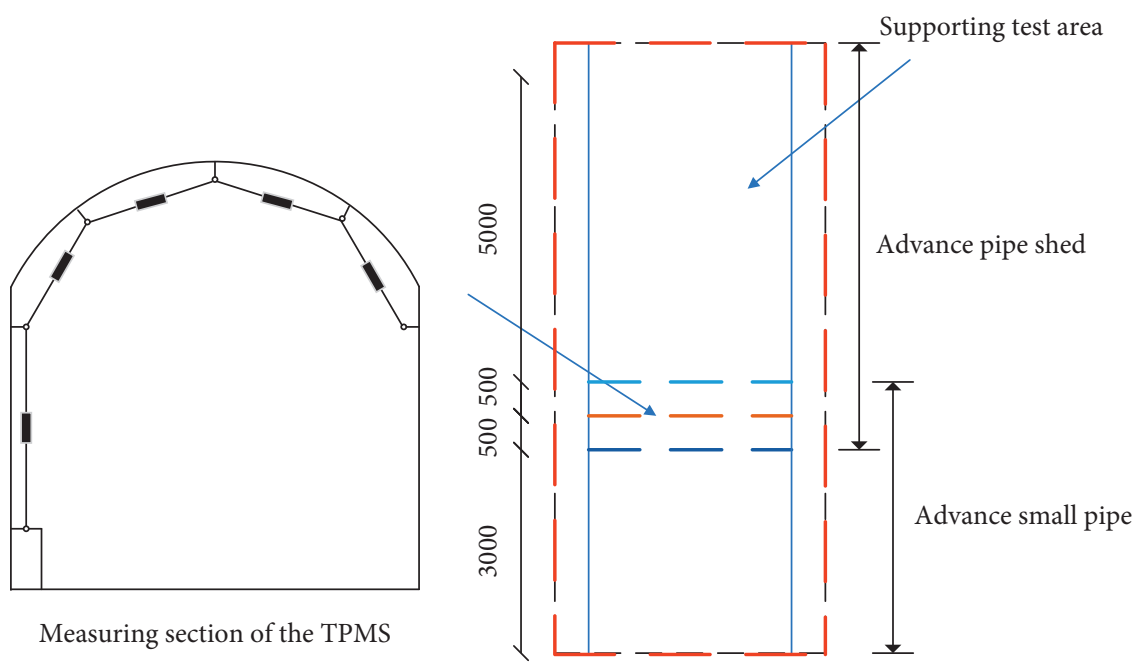

Figure 11: Schematic diagram of measuring section location (unit: $\mathrm{mm}$ ).

top was conducted for this area, with the length set as $6 \mathrm{~m}$. After the pipe shed section was tunneled $5 \mathrm{~m}, 4 \mathrm{~m}$ of the advanced small pipe support in the arch top was set up forward. When the advanced small pipe support was completed, tunneling stopped after $3 \mathrm{~m}$ of forward construction. Afterwards, the mesh shotcrete and the steel arch supports were installed with an arch spacing of $0.5 \mathrm{~m}$. After the shotcrete was completed, the TPMS was installed, and the TPMS was located at the junction between the advance pipe shed and the advance small pipe, where the support was relatively weak.

As shown in Figure 12, the chamber span was $4.0 \mathrm{~m}$, the height of the arch apex was $3.0 \mathrm{~m}$, and the straight-wall section had a three-centered arch shape. There were 6 measuring points arranged in the measuring section with an open loop system. Except for measuring point 0, which was set on the ground as a reference point, the other 5 measuring points were all arranged on the arch, with measuring point 3 at the apex of the arch, points 1 and 5 in the arch foot, and points 2 and 4 located on the hance.

3.3. Section Optimization and Coordinate Transformation. According to the optimization and transformation method described in Section 2.4, the optimal plane corresponding to each measuring point of the in situ monitoring test is solved, and the initial 2D coordinates of each point are established.

By total station measurements, the initial 3D coordinates in the geodetic coordinate system of each measuring point in the field test are shown in Table 2. As shown in Table 2, the numerical values of the measuring points in $3 \mathrm{D}$ geodetic coordinates are all relatively great, and numerical overflow may occur during optimization. However, the origin position of the geodetic coordinate system does not affect the obtainment of the optimal plane and coordinate transformation. Therefore, the geodetic coordinate system origin is 


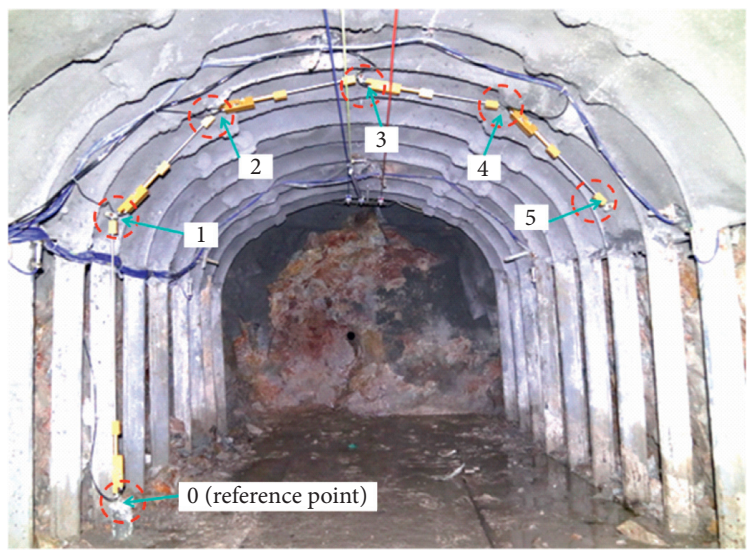

Figure 12: Distribution of measuring points.

TABLE 2: Initial 3D coordinates of TPMS measuring points.

\begin{tabular}{lccccc}
\hline Measuring point & $X(\mathrm{~m})$ & $Y(\mathrm{~m})$ & $Z(\mathrm{~m})$ & $X_{d}(\mathrm{~m})$ & $Y_{d}(\mathrm{~m})$ \\
\hline 0 & 4524349.584 & 360613.163 & 1606.893 & 1.584 & 3.163 \\
1 & 4524349.582 & 360613.173 & 1608.374 & 1.582 & 3.173 \\
2 & 4524349.399 & 360612.651 & 1608.933 & 1.399 & 2.651 \\
3 & 4524349.137 & 360611.909 & 1609.084 & 1.137 & 2.374 \\
4 & 4524348.889 & 360611.183 & 1608.920 & 0.889 & 1.909 \\
5 & 4524348.688 & 360610.693 & 1608.309 & 0.688 & 1.183 \\
\hline
\end{tabular}

translated to point $(4524348,360610,1606)$, and the corresponding initial $3 \mathrm{D}$ coordinates of each measuring point are transformed into $\left(X_{d}, Y_{d}, Z_{d}\right)$ coordinates. According to the objective function for equation (13), the optimal monitoring plane determined by all the measuring points is calculated. When parameters $a 0, a 1$, and $a 2$ in equation (12) are multiplied by any nonzero constant that can still meet the equation, only the proportional relations between the parameters of the plane equations need to be obtained. Therefore, set $a 1=1$ and the Levenberg-Marquardt (LM) integration algorithm and particle swarm optimization (PSO) may be used, where LM is a least squares fitting algorithm and PSO is an iterative optimization algorithm. Via calculations using MATLAB software, the plane parameters $a 0=-0.4629$ and $a 2=-0.3531$ can be obtained. Then, equation (14) is solved, and the $3 \mathrm{D}$ coordinate of the projection point for the reference point in the optimal monitoring plane is $(1.5802541,3.1643227,0.893)$. Finally, according to equation (15), the $2 \mathrm{D}$ coordinate of each measuring point on the optimal monitoring plane $(x, y)$ can be obtained (Table 3).

3.4. Analysis of the Long-Term Monitoring Results. After installation and debugging of the test instruments and with December 18th, 2016, as the start time for monitoring, 561 days of test data were obtained by July 2nd, 2018. As convergence and deformation monitoring was mainly focused on local variations in the section, in subsequent data processing, the displacement of the reference point was returned to zero, and the relative displacement of each monitoring point was obtained. Time-history curves for the $x$ and $y$ directions and the total displacement of each measuring point relative to the reference point are shown in Figure 13.

As shown in Figure 13, in the initial monitoring stage, the total displacement of each measuring point increased almost linearly with time, and the displacement increase rate of each measuring point showed a tendency to significantly decrease around the 30th day, and the displacement increased slowly after the 30th day and showed a tendency to converge to fixed values. The monitoring data of the surrounding rock indicated the following phenomena: there was mainly a horizontal displacement at measuring points 1 and 5, while the vertical displacement was small. In contrast, a vertical displacement mainly occurred, while the horizontal displacement could be ignored in the case of point 3 . Because of the symmetrical location of measuring points 2 and 4 , their displacement variation laws were similar. The horizontal displacement of the tunnel was larger than the vertical displacement, mainly because the tunnel was located in the Shiyuejing Fault zone, the rock lithology was poor, the in situ stress condition was complex, and the tunnel vault was presupported. The surrounding rock deformation was mainly controlled by the horizontal stress.

The direction of the total displacement of each measuring point can be obtained by the ratio of the displacement value in the $x$ direction to the displacement value in the $y$ direction:

$$
\tan \varphi=\frac{\mathrm{d}_{y}}{\mathrm{~d}_{x}},
$$

where $\varphi$ is the angle between the displacement of the measuring point and the horizontal direction and $\mathrm{d}_{x}$ and $\mathrm{d}_{y}$ 
TABLE 3: $2 \mathrm{D}$ coordinates of each measuring point in the optimal plane.

\begin{tabular}{lcc}
\hline Measuring point & $x(\mathrm{~m})$ & $y(\mathrm{~m})$ \\
\hline 0 & 0 & 0 \\
1 & -0.00826 & 1.481 \\
2 & 0.51332 & 2.040 \\
3 & 1.25532 & 2.191 \\
4 & 1.97870 & 2.027 \\
5 & 2.47749 & 1.416 \\
\hline
\end{tabular}

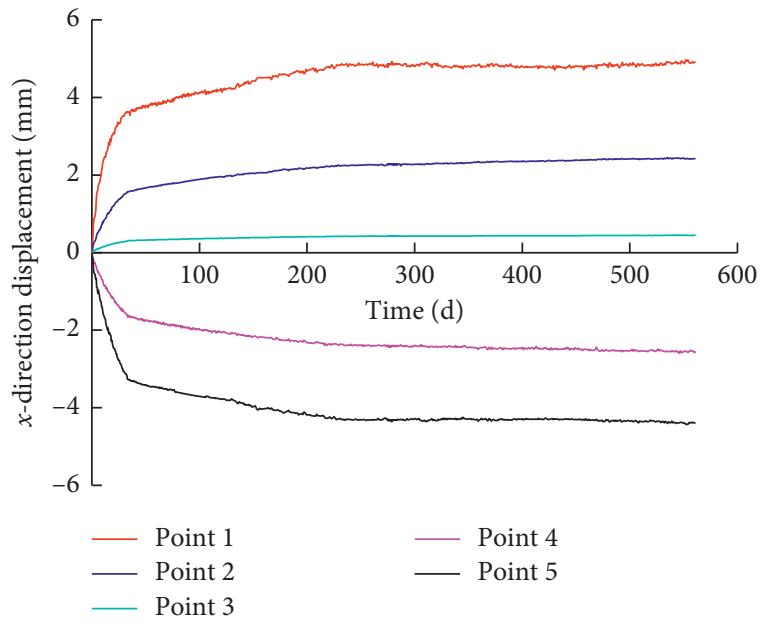

(a)

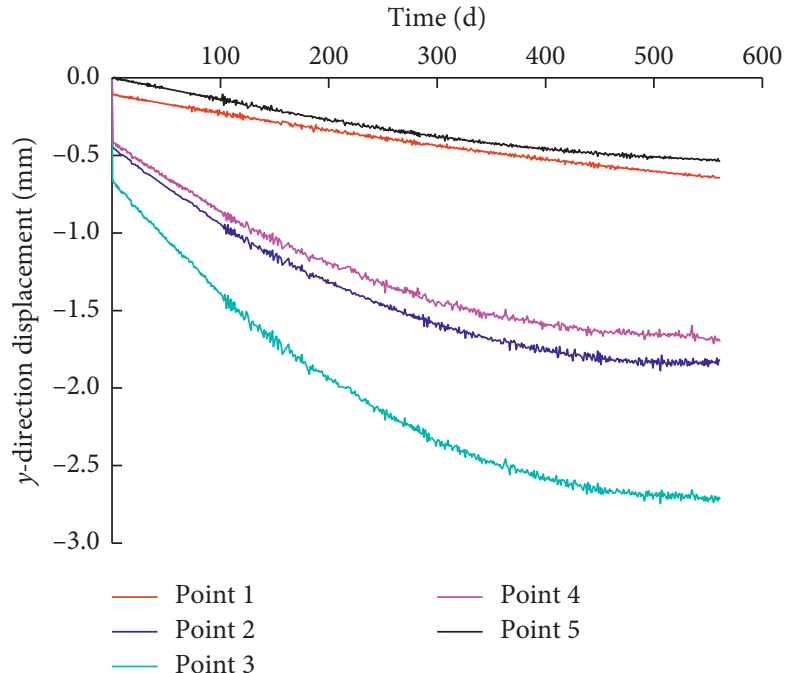

(b)

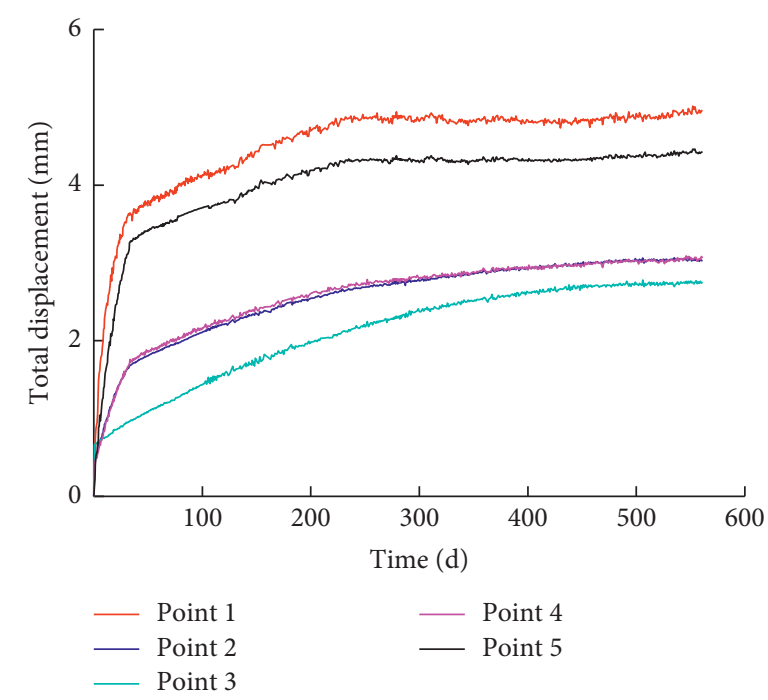

(c)

FIGURE 13: Time-history curves of the displacement of each measuring point: (a) $x$-direction displacement; (b) $y$-direction displacement; (c) total displacement.

are the displacement values of the measuring point in the horizontal and vertical directions, respectively.

According to equation (16) and the data of Figure 13, the displacement direction angle of each measuring point at different times could be obtained, as shown in Table 4.
Based on the obtained displacement direction angle, the displacement trend diagrams of the measurement section at 30, 200, 400, and 561 days after monitoring were given, that is, the displacement envelope diagram, as shown in Figure 14. 
TABLE 4: Displacement direction angle of each measuring point at different times.

\begin{tabular}{lcccccccccccc}
\hline \multirow{2}{*}{ Measuring point } & \multicolumn{3}{c}{$30 \mathrm{~d}$} & \multicolumn{3}{c}{$200 \mathrm{~d}$} & \multicolumn{3}{c}{$400 \mathrm{~d}$} & \multicolumn{3}{c}{$561 \mathrm{~d}$} \\
& $\mathrm{~d}_{x}(\mathrm{~mm})$ & $\mathrm{d}_{y}(\mathrm{~mm})$ & $\varphi\left({ }^{\circ}\right)$ & $\mathrm{d}_{x}(\mathrm{~mm})$ & $\mathrm{d}_{y}(\mathrm{~mm})$ & $\varphi\left({ }^{\circ}\right)$ & $\mathrm{d}_{x}(\mathrm{~mm})$ & $\mathrm{d}_{y}(\mathrm{~mm})$ & $\varphi\left({ }^{\circ}\right)$ & $\mathrm{d}_{x}(\mathrm{~mm})$ & $\mathrm{d}_{y}(\mathrm{~mm})$ & $\varphi\left({ }^{\circ}\right)$ \\
\hline 1 & 3.514 & 0.148 & 2.41 & 4.683 & 0.344 & 4.20 & 4.802 & 0.532 & 6.32 & 4.914 & 0.645 & 7.48 \\
2 & 1.496 & 0.611 & 22.22 & 2.174 & 1.324 & 31.34 & 2.339 & 1.766 & 37.05 & 2.425 & 1.820 & 36.89 \\
3 & 0.281 & 0.907 & 72.79 & 0.408 & 1.940 & 78.12 & 0.431 & 2.587 & 80.54 & 0.445 & 2.716 & 80.70 \\
4 & 1.521 & 0.565 & 20.38 & 2.318 & 1.192 & 27.21 & 2.439 & 1.587 & 33.05 & 2.546 & 1.701 & 33.75 \\
5 & 3.064 & 0.040 & 0.75 & 4.183 & 0.268 & 3.67 & 4.280 & 0.453 & 6.04 & 4.393 & 0.536 & 6.96 \\
\hline
\end{tabular}

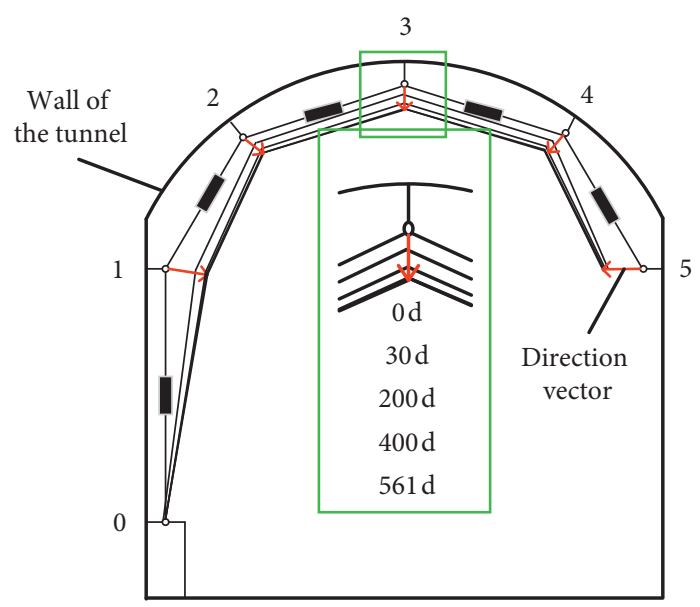

FIGURE 14: Displacement envelope diagrams and direction vector of the displacements.

\section{Discussion and Suggestions}

4.1. Comparative Analysis of the TPMS and Artificial Convergence. The TPMS is a new-type convergence displacement monitoring device. To verify the feasibility and accuracy of monitoring results, especially after TPMS installation is completed, the convergence displacement is measured manually using a convergence gauge. The results are compared and analyzed with the TPMS data. The convergence gauge is a $30 \mathrm{~m}$ digital convergence gauge manufactured by Jiangsu Haiyan Engineering Material and Instrument Co., Ltd. The horizontal measuring line is the connected line between measuring points 1 and 5 on the TPMS; the measurement time lasts 17 days, from December 18th, 2016 to January 3rd, 2017. The relative displacement of measuring points 1 and 5 of the TPMS in the horizontal direction is just the convergence displacement of the 1-5 measuring line. The measurement results from the TPMS and artificial convergence monitoring are shown in Figure 15.

A linear function is used to conduct a linear fitting on the TPMS and manually measured data, and the fitting equations are

$$
\begin{aligned}
& y_{1}=0.27297 t+0.80094 \\
& y_{2}=0.28189 t+0.7726
\end{aligned}
$$

where $y_{1}$ denotes the measured results from the TPMS, $y_{2}$ denotes the measured results from the artificial convergence gauge, and $t$ denotes the measurement time. The measured data from the TPMS are in better agreement with those from the artificial convergence gauge, verifying the accuracy of the TPMS data and the feasibility of using the TPMS as a newtype monitoring method for tunnel section convergence.

Compared with traditional convergence monitoring methods, such as the convergence meter, the TPMS can realize the continuous monitoring, automatic collection and transmission of monitoring data, and remote access whenever necessary, without affecting the transportation in the tunnel. Furthermore, the monitoring frequency of the TPMS can be set freely according to the need. The measurement accuracy of the TPMS relates to the accuracy of the tilt sensor and displacement sensor, which reach $0.003^{\circ}$ and $0.01 \mathrm{~mm}$, respectively.

4.2. Comparative Analysis of the TPMS and BCS. As a representative convergence measurement system, the BCS measurement schematic diagram is shown in Figure 16. From Figures 1 and 16, similarities are evident between the TPMS and the BCS, such as the structure of the measurement system and relative displacement measurement between two measuring points. However, the measurement principles of the TPMS and BCS are clearly different; the BCS obtains the relative displacement of adjacent measuring points by two tilt sensors and the length of the measuring arm, and the TPMS obtains the relative displacement of adjacent measuring points through tilt and displacement sensors. Compared with the BCS, the TPMS integrates both the displacement sensor and the tilt sensor so that only one 


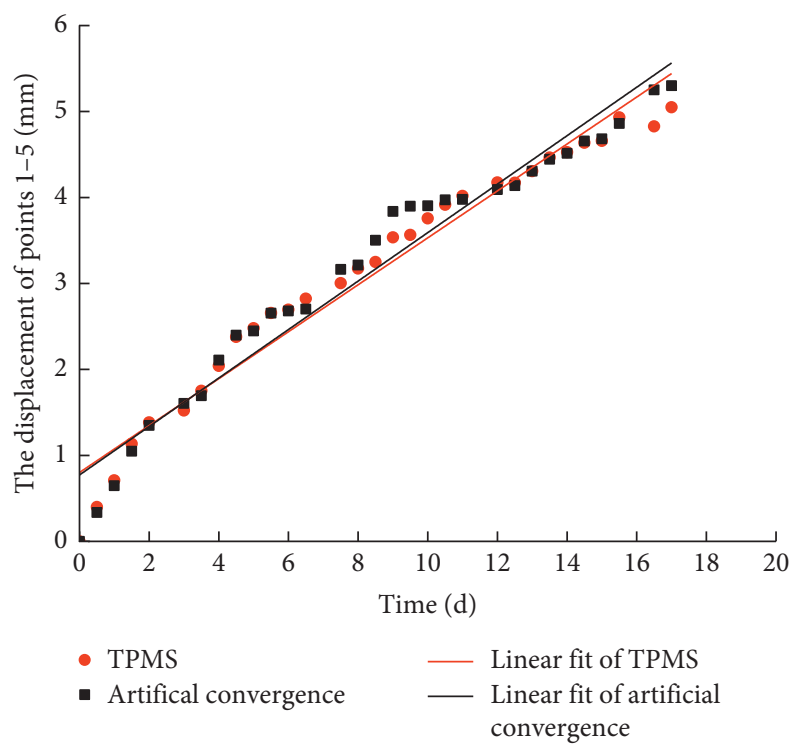

FIGURE 15: Data diagram of the TPMS and artificial convergence monitoring.

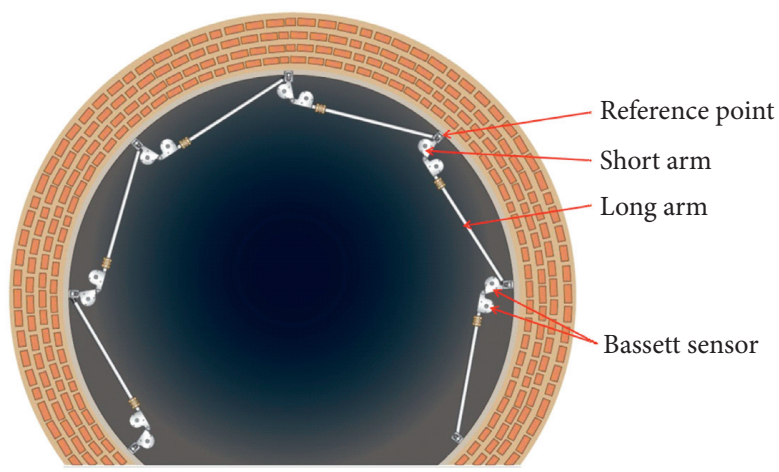

FIgURE 16: Measurement schematic diagram of the BCS.

measuring arm can transmit the angle and displacement between two measuring points. Therefore, the TPMS simplifies the long arm and short arm structure of the BCS and avoids the errors introduced in calculating the displacement by using a fixed length long arm and short arm, thus forming a simple and effective measurement method for the convergence displacement of the tunnel.

4.3. Suggestions for the TPMS. Through the practical application of in situ monitoring, the following three suggestions for improvement are summarized. First, the long-term stability of the displacement sensor used in the TPMS monitoring unit is worse than that of the tilt sensor, and data floating sometimes occurs when the acquisition frequency is dense. If the displacement sensor is changed from the voltage sensor to the vibrating-wire sensor, the error caused by the sensor itself can be reduced. Second, although the open loop structure can reduce interference in the use of the tunnel, the monitoring configuration should be made up of a closed loop structure so that the closure error can be effectively reduced. Third, to obtain the absolute deformation of each measuring point, it is necessary to regularly measure the spatial coordinates of the reference point through engineering datum point guidance. In addition, since this project monitors the tunnel after the support, the displacement is small. To ensure the accuracy of the measurement, we chose a small range of the sensor. When monitoring a large deformation tunnel, it is necessary to select a sensor of the appropriate range according to the actual situation.

\section{Conclusions}

A new contact TPMS was introduced in detail, and the method of obtaining the optimal monitoring plane and converting coordinates of the measuring points was established to eliminate the actual installation and positioning deviation of the monitoring system. The displacement calculation formulas of the tunnel cross section measuring points based on the two-dimensional plane coordinate system were proposed, providing a theoretical basis for the popularization and application of this monitoring system. 
Through long-term monitoring of the convergence displacement of the surrounding rock in the BET, the timehistory curves of each measuring point and displacement envelope diagrams for the measurement section were obtained. Monitoring application practice and results show that the TPMS can realize continuous monitoring, automatic collection and transmission of monitoring data, remote access whenever necessary, without affecting the transportation in the tunnel, and high accuracy, which can reach $0.01 \mathrm{~mm}$, and can be applied to the long-term health monitoring of the lining or envelope of the tunnel and underground engineering.

The reliability of TPMS monitoring is verified by comparing with the monitoring data of a convergence meter. To better apply the TPMS, three further research directions for improvement are proposed. Additionally, the convergence monitoring method such as the TPMS and the BCS can be used in the same measurement section for comparative analysis.

\section{Abbrevations}

$x, y: \quad$ The initial 2D coordinates of measuring point $(\mathrm{m})$

$X, Y, Z$ : The initial 3D coordinates of measuring point $(\mathrm{m})$

$\Delta \theta_{i j}: \quad$ The rotation angle of the measuring arm $\left(^{\circ}\right)$

$\Delta L_{i}$ : The length variation $h$ of the measuring arm (m)

$\theta: \quad$ The inclination of the tilt sensor $\left({ }^{\circ}\right)$

$L: \quad$ The displacement of the displacement sensor $(\mathrm{m})$

$V_{t}: \quad$ The output voltage of the tilt sensor $(\mathrm{V})$

C: $\quad$ The calibration constants for the tilt sensor

$V_{d}$ : The output voltage of the displacement sensor (V)

$K_{d}$ : The calibration constants for the displacement sensor $(\mathrm{mm} / \mathrm{V})$

$b$ : The calibration constants for the displacement sensor (mm)

$a_{0}, a_{1}$, The plane equation parameters

$a_{2}$ :

$\mathrm{d}_{x}, \mathrm{~d}_{y}$ : The displacement values of the measuring point in the horizontal and vertical directions

$\varphi$ : $\quad$ The angle between the displacement of the measuring point and the horizontal direction

$t: \quad$ Time.

\section{Data Availability}

The data used to support the findings of this study are included within the article.

\section{Conflicts of Interest}

The authors declare that they have no conflicts of interest regarding the publication of this paper.

\section{Acknowledgments}

The authors wish to extend their sincere thanks for the support from the China Atomic Energy Authority (CAEA) for China's URL Development Program and the Geological Disposal Program. This work was supported by the China
Atomic Energy Authority (grant no. FZ1402) and National Natural Science Foundation of China (grant no. 51408605).

\section{References}

[1] P. P. Oreste and D. Dias, "Stabilisation of the excavation face in shallow tunnels using fibreglass dowels," Rock Mechanics and Rock Engineering, vol. 45, no. 4, pp. 499-517, 2012.

[2] L. Qiao, S. Li, Z. Wang, H. Tian, and L. Bi, "Geotechnical monitoring on the stability of a pilot underground crude-oil storage facility during the construction phase in China," Measurement, vol. 82, pp. 421-431, 2016.

[3] S. Yang, W. Liu, H. Shi, and F. Huang, "A study on the theory and method of non-contact monitoring for tunnel rock deformation based on free stationing of a total station," China Civil Engineering Journal, vol. 39, no. 4, pp. 100-104, 2006.

[4] A. Berberan, M. Machado, and S. Batista, "Automatic multi total station monitoring of a tunnel," Survey Review, vol. 39, no. 305, pp. 203-211, 2007.

[5] Y. Luo, J. Chen, W. Xi et al., "Application of a total station with RDM to monitor tunnel displacement," Journal of Performance of Constructed Facilities, vol. 31, no. 4, 2017.

[6] S. Slob and R. Hack, "3D terrestrial laser scanning as a new field measurement and monitoring technique," in Engineering Geology for Infrastructure Planning in Europe: A European Perspective, H. Robert, A. Rafig, and C. Robert, Eds., pp. 179-189, Springer, Berlin, Germany, 2004.

[7] X. Xie and X. Lu, "Development of a 3D modeling algorithm for tunnel deformation monitoring based on terrestrial laser scanning," Underground Space, vol. 2, no. 1, pp. 16-29, 2007.

[8] G. Walton, D. Delaloye, and M. S. Diederichs, "Development of an elliptical fitting algorithm to improve change detection capabilities with applications for deformation monitoring in circular tunnels and shafts," Tunnelling and Underground Space Technology, vol. 43, pp. 336-349, 2014.

[9] Y.-H. Li, S.-D. Xu, and J.-P. Liu, "A new convergence monitoring system for tunnel or drift based on draw-wire displacement sensors," Tunnelling and Underground Space Technology, vol. 49, pp. 92-97, 2015.

[10] T. Tao and H. Wang, "Modelling of longitudinal evolutionary power spectral density of typhoon winds considering highfrequency subrange," Journal of Wind Engineering and Industrial Aerodynamics, vol. 193, Article ID 103957, 2019.

[11] T. Tao, Y. L. Xu, and Z. F. Huang, "Buffeting analysis of longspan bridges under typhoon winds with time-varying spectra and coherences," Journal of Structural Engineering, vol. 146, no. 2, Article ID 4020255, 2020.

[12] T. Tao, P. Shi, and H. Wang, "Spectral modelling of typhoon winds considering nexus between longitudinal and lateral components," Renewable Energy, vol. 162, pp. 2019-2030, 2020.

[13] R. H. Bassett, J. P. Kimmance, and C. Rasmussen, “An automated electrolevel deformation monitoring system for tunnels," Proceedings of the Institution of Civil Engineers Geotechnical Engineering, vol. 137, no. 3, pp. 117-125, 1999.

[14] L. Simeoni and L. Zanei, "A method for estimating the accuracy of tunnel convergence measurements using tape distometers," International Journal of Rock Mechanics and Mining Sciences, vol. 46, no. 4, pp. 796-802, 2009.

[15] W. D. Li, N. Lin, and X. Chen, "Research on 3D tunnel modeling based on close-range photogrammetry," Advanced Materials Research, vol. 1076, pp. 1934-1940, 2014.

[16] F. Ariznavarreta-Fernández, C. González-Palacio, A. MenéndezDíaz, and C. Ordoñez, "Measurement system with angular 
encoders for continuous monitoring of tunnel convergence," Tunnelling and Underground Space Technology, vol. 56, pp. 176-185, 2016.

[17] Q. Liu, J. Chen, P. Huang, X. Pu, Y. Zhao, and X. Yuan, "Multivariate information dynamic monitoring system application of tunnel construction process model test," Electronic Journal of Geotechnical Engineering, vol. 21, no. 9, pp. 3519-3533, 2016.

[18] S.-Q. Sun, S.-C. Li, L.-P. Li, S.-S. Shi, Z.-Q. Zhou, and C.-L. Gao, "Design of a displacement monitoring system based on optical grating and numerical verification in geomechanical model test of water leakage of tunnel," Geotechnical and Geological Engineering, vol. 36, no. 4, pp. 2097-2108, 2018.

[19] J. Wang, L. Chen, R. Su, and X. Zhao, "The Beishan underground research laboratory for geological disposal of highlevel radioactive waste in China: planning, site selection, site characterization and in situ tests," Journal of Rock Mechanics and Geotechnical Engineering, vol. 10, no. 3, pp. 411-435, 2018.

[20] J. Wang, E. Li, L. Chen et al., "Measurement and analysis of the internal displacement and spatial effect due to tunnel excavation in hard rock," Tunnelling and Underground Space Technology, vol. 84, pp. 151-165, 2019. 